2. Deschamps C, Tirnaksiz BM, Darbandi R, Trastek VF, Allen MS, Miller DL, et al. Early and long term results of prosthetic chest wall reconstruction. J Thorac Cardiovasc Surg. 1999;117:588-92.

3. Weyant MJ, Bains MSD, Venkatraman E, Downey RJ, Park BJ, Flores RM, et al. Results of chest wall resection and reconstruction with and without rigid prosthesis. Ann Thorac Surg. 2006;81:279-85.
4. Garcia-Tutor E, Yeste L, Murillo J, Aubà C, Sanjulian M, Torre W. Chest wall reconstruction using iliac bone allografts and muscle flaps. Ann Plast Surg. 2004;52:54-60.

5. Fisher S, de Perrot M, Sekine Y, Keshavjee S. Long term survival after multiple resections of a fibrosarcoma involving the lung and the chest wall. Eur J Cardiothorac Surg. 2001;20:421-3.

\title{
Mediastinal mature teratoma with complex rupture into the pleura, lung, and bronchus complicated with mycoplasma pneumonia
}

Ching-Wen Yu, MD, ${ }^{a}$ Ming-Jang Hsieh, MD, ${ }^{\mathrm{b}}$ Kao-Pin Hwang, MD, ${ }^{c}$ Chung-Cheng Huang, MD, ${ }^{d}$ Shu-Hang Ng, MD, ${ }^{d}$ and Sheung-Fat Ko, MD, ${ }^{d}$ Kaohsiung, Taiwan

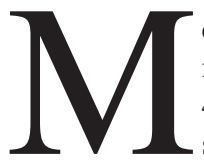

ediastinal mature teratoma (MMT) is an uncommon neoplasm in children, accounting for only $4.3 \%$ of all germ cell tumors. ${ }^{1-3}$ It may cause stridor and dyspnea by direct tracheal compression in children $<2$ years old but is rarely symptomatic in later childhood. ${ }^{1}$ Occasionally, MMT may rupture into the pleural cavity, pericardium, lung, and tracheobronchial tree, leading to protean clinical presentations. ${ }^{1-5}$ To our knowledge, cystic MMT with complex rupture into the pleura, lung, and bronchus complicated with mycoplasma pneumonia has not previously been described. We report such a rare case in a 13-year-old boy with an emphasis on the perplexing clinical and computed tomographic (CT) features and the importance of early surgical treatment.

\section{Clinical Summary}

A 13-year-old boy suffered from severe paroxysmal cough with brownish fluid expectorant and mild fever for 10 days. He visited a community hospital where the chest radiograph revealed left lower lung consolidation with effusion, and CT suggested pneumonia with necrotic changes and empyema. $\mathrm{He}$ was treated for lobar pneumonia. Despite antibiotic treatment, cough and fever persisted. He was then referred to our hospital. Physical examination revealed an acutely ill-looking patient with fever $\left(39^{\circ} \mathrm{C}\right)$ and decreased but coarse breathing sounds over the left lower chest. Abnormal laboratory data included a white blood cell count of $12,300 / \mathrm{mm}^{3}$ with $77 \%$ polymorphonuclear leukocytes and elevated C-reactive protein (129 mg/L). Serum cold-agglutinin titer of 1:32 and positive mycoplasma antibody tests (immunoglobulin $\mathrm{G}=21 \mathrm{BU} / \mathrm{mL}$, immunoglobulin $\mathrm{M}=28.1 \mathrm{BU} / \mathrm{mL}$; normal $<10 \mathrm{BU} / \mathrm{mL}$ ) were also noted. Chest radiograph showed left lower lung opacity and left pleural effusion. Chest tapping harvested $75 \mathrm{~mL}$ mildly brownish fluid with slight turbidity but culture was negative. Mycoplasma pneumonia was suspected. After 1-week antibiotic treatment, the fever subsided. However, severe paroxysmal cough with intermittent brownish fluid expectorant was still noted. Follow-up radiograph revealed persistent left lower lung opacity. Chest CT (Figure 1) revealed a heterogeneous, multiloculated, cystic mass in the left anterior mediastinum with a small fatty focus, highly suggestive of MMT. Perforation into the adjacent pleural cavity, lingular segment, and bronchus and focal oblit-
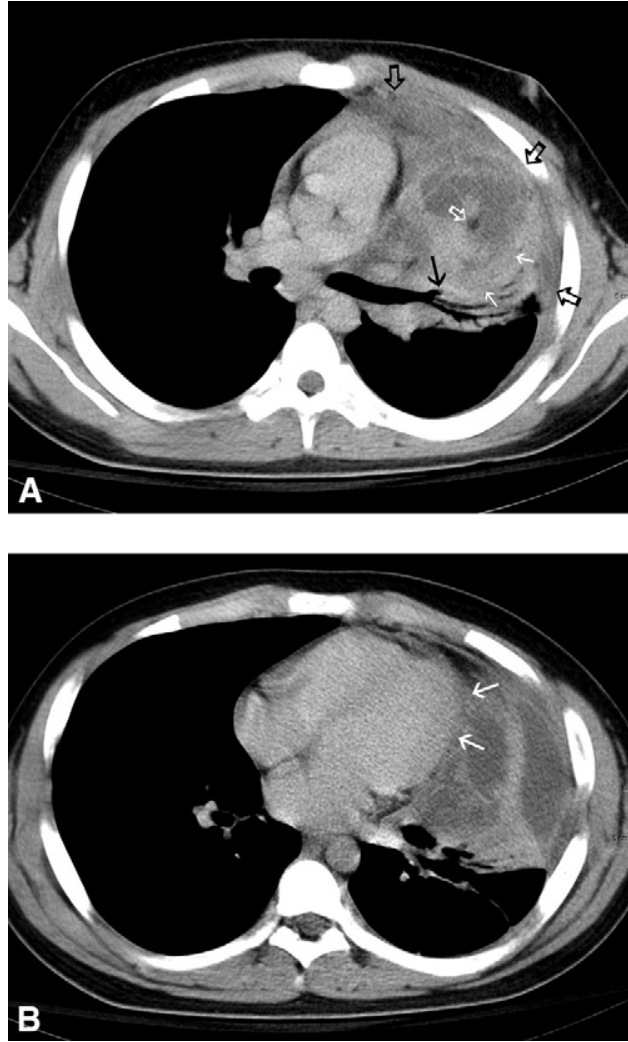

Figure 1. A, Axial contrast-enhanced CT showing a heterogeneous, multiloculated, cystic mass in the left anterior mediastinum with a small fatty focus (open white arrow) perforating into the pleura (black open arrows), lingular lobe (small white arrows), and lingular bronchus (black arrow). B, CT at the midventricular level showing obliteration of the pericardial fat (large white arrows), suggesting close attachment of the pericardium by the tumor. Minimal pericardial effusion is also noted.

eration of adjacent pericardial fat were also demonstrated. Serum alpha-fetoprotein, beta-human chorionic gonadotrophin, 
and carcinoembryonic antigen (CEA) levels were normal. At thoracotomy, the surgeon confirmed the imaging findings and further found a focal erosion with impending rupture of the pericardium. Total tumor resection, thymectomy, extrapleural pneumonolysis, pleural decortication, lingular lobectomy, and partial left lower lobe resection were performed. Pathologic examination confirmed that MMT had ruptured into the pleura, lingular lobe, and bronchus. The levels of amylase, lipase, and CEA in the tumor content were not elevated. After surgery, the patient recovered uneventfully and remained well at 3-year follow-up.

\section{Discussion}

MMT is a benign, slow-growing tumor within or near the thymus gland typically affecting 20 - to 40 -year-old adults. ${ }^{2,3}$ MMT is generally asymptomatic. Occasionally, it may rupture into the mediastinum, pleura, lung, bronchus, and pericardium, with various clinical presentations including chest and/or back pain, fever, cough with hairy and/or sebaceous expectorant, and even cardiac tamponade. ${ }^{1-5}$ Ruptured MMT among pediatric populations is rare. ${ }^{1}$ In our patient, clinical presentations of fever, paroxysmal severe cough, positive cold-agglutinin titer, elevated mycoplasma antibody levels, and radiographic features of pulmonary consolidation and effusion were indicative of coexistent mycoplasma pneumonia that, in turn, masked the underlying ruptured MMT. In retrospect, persistent severe paroxysmal cough with fluid expectorant similar to that yielded from chest tapping might suggest MMT with rupture to the bronchus.

CT is the modality of choice for evaluating MMT, which typically manifests as a heterogeneous mass containing soft tissue, fat, fluid, and/or calcium. However, as seen in our case, MMT may mimic pneumonia when complicated with rupture and/or infection. ${ }^{3}$ Scrutiny of any intralesional fat component can help establish diagnosis, but $15 \%$ of MMT may be purely cystic without fat or calcium. ${ }^{2,3}$ Although rare, this particular case demonstrates that MMT might present with complex rupture involving the pleura, lung, and bronchus and even pericardial erosion with impending rupture. The mechanism of MMT rupture remains undetermined. High levels of digestive enzymes in the tumor content may contribute to rupture into adjacent structures. ${ }^{4}$ However, in our case, lack of elevation of the intratumoral amylase, lipase, and CEA levels supported the other hypothesis that chemical inflammation, ischemia, pressure necrosis, and/or infection may trigger tumor rupture. ${ }^{3,4}$ Once diagnosed, early surgery of MMT is recommended. Delayed surgical resection of ruptured MMT is more complicated due to its digestive autolysis and chemical, ischemic, and/or necrotic sequelae. $^{3,4}$ Fortunately, successful surgical treatment was achieved in our patient before MMT eroded into the pericardium with potentially life-threatening acute cardiac tamponade. $^{5}$

\section{References}

1. Matsubara K, Aoki M, Okumura N, Menju T, Nigami H, Harigaya H, et al. Spontaneous rupture of mediastinal cystic teratoma into the pleural cavity: report of two cases and review of the literature. Pediatr Hematol Oncol. 2001;18:221-7.

2. Moeller KH, Rosado-de-Christenson ML, Templeton PA. Mediastinal mature teratoma: imaging features. AJR Am J Roentgenol. 1997;169: 985-90.

3. Sasaka K, Kurihara Y, Nakajima Y, Seto Y, Endo I, Ishikawa T, et al. Spontaneous rupture: a complication of benign mature teratomas of the mediastinum. AJR Am J Roentgenol. 1998;170:323-8.

4. Ege G, Akman H, Cakiroglu G, Kalayci G. Spontaneous rupture of mediastinal cystic teratoma with high levels of amylase, lipase, CA 19-9, CA 125 and CEA in cystic fluid: a case report. Acta Radiol. 2004;45:111-2

5. Oomman A, Santhosham R, Vijayakumar C, Jayaraman S, Ramachandran P, Kumar S. Anterior mediastinal teratoma presenting as cardiac tamponade. Indian Heart J. 2004;56:64-6. 